\title{
LINEAR INDEPENDENCE OF TRANSLATIONS
}

\author{
JOSEPH ROSENBLATT
}

(Received 28 July 1992)

Communicated by P. G. Dodds

\begin{abstract}
It was shown by Edgar and Rosenblatt that if $f \in L_{p}\left(\mathbb{R}^{n}\right), 1 \leq p<2 n /(n-1)$, and $f \neq 0$, then $f$ has linearly independent translates. Using a result of Hörmander, it is shown here that the same theorem holds if $p=2 n /(n-1)$. This gives a sharp result because for $n \geq 2$, there exists $f \in C_{0}\left(\mathbb{R}^{n}\right), f \neq 0$, which is simultaneously in all $L_{p}\left(\mathbb{R}^{n}\right), p>2 n /(n-1)$, that has a linear dependence relation among its translates. References and some discussion are included.
\end{abstract}

1991 Mathematics subject classification (Amer. Math. Soc.): 39A10, 42A38.

Given $f \in L_{p}\left(\mathbb{R}^{n}\right)$ where $1 \leq p \leq \infty$, and $t \in \mathbb{R}^{n},{ }_{t} f$ is the translation defined by ${ }_{t} f(x)=f(x-t)$ for all $x \in \mathbb{R}^{n}$. A given $f$ has linearly independent translations if and only if for all distinct vectors $t_{1}, \ldots, t_{m} \in \mathbb{R}^{n}$, and non-zero complex numbers $a_{1}, \ldots, a_{m}$, the linear combination $\sum_{i=1}^{m} a_{i t_{i}} f$ is not zero. The purpose of this note is to complete a theorem in Edgar and Rosenblatt [1]. It was shown there that if $1 \leq p<2 n /(n-1)$, then every non-zero $f \in L_{p}\left(\mathbb{R}^{n}\right)$ has linearly independent translations. It is also remarked that, for $n \geq 2$, there exists a $C_{0}\left(\mathbb{R}^{n}\right)$ function $f$ that does not have linearly independent translations, which is also in all $L_{p}\left(\mathbb{R}^{n}\right)$ for $p>2 n /(n-1)$. But furthermore it happens that an earlier theorem in Hörmander [3] shows that for the critical index $p=2 n /(n-1), n \geq 2$, any non-zero function in $L_{p}\left(\mathbb{R}^{n}\right)$ has linearly independent translations. See also Guo [2].

LEMMA 1. If $f \in L_{p}\left(\mathbb{R}^{n}\right)$, where $2<p \leq 2 n /(n-1)$ and $p<\infty$, then $\int_{\{R<|x|<2 R\}}|f(x)|^{2} d x=o(R)$ as $R \rightarrow \infty$.

PROOF. As in [3, Corollary 3.3], this follows from Hölder's inequality.

(C) 1995 Australian Mathematical Society 0263-6115/95 \$A2.00+0.00

Partially supported by NSF Grant 8802126 
THEOREM 2. (Hörmander [3]) Let $u$ be a tempered distribution supported by a real analytic set $A$ of codimension $k>0$, and assume that $\hat{u} \in L_{\mathrm{loc}}^{2}\left(\mathbb{R}^{n}\right)$. Assume that for every analytic manifold $M$ contained in $A$ and every $x_{0} \in M$, there is an open cone $\Gamma \subset \mathbb{R}^{n}$ which contains some normal of $M$ at $x_{0}$, and such that on $\Gamma_{R}=\{x \in \Gamma: R<|x|<2 R\}$,

$$
\lim _{R \rightarrow \infty} R^{-k} \int_{\Gamma_{R}}|\hat{u}(x)|^{2} d x=0 .
$$

Then $u=0$.

PROOF. See [3, Theorem 2.4] and the remark following it.

THEOREM 3. For any non-zero $f \in L_{p}\left(\mathbb{R}^{n}\right)$, where $1 \leq p \leq 2 n /(n-1)$ and $p<\infty, f$ has linearly independent translations.

PROOF. As in [1, Theorem 1.2], this result follows for $1 \leq p \leq 2$ for general locally compact abelian groups without non-trivial compact subgroups by a standard argument with Fourier transforms. So we may assume that $2<p \leq 2 n /(n-1), p<\infty$. This means that $f$ is in $L_{\text {loc }}^{2}\left(\mathbb{R}^{n}\right)$ also. Suppose that $f$ does not have linearly independent translates. Then the Fourier transform $\hat{f}$ of $f$ as a tempered distribution will have support in a real analytic set which is the zero set of some non-zero trigonometric polynomial. Let $u=\hat{f}$ in Theorem 2 . By Lemma $1, u$ satisfies the growth condition required in Theorem 2 for every cone $\Gamma$. Therefore $u=0$ and so $f=0$.

REMARKS. (a) When $n=1$, there is a companion theorem in [1] to the above that says that all non-zero functions in $C_{0}(\mathbb{R})$ have linearly independent translations. This is also proved by using Fourier transforms of tempered distributions. There ought to be a proof of this result, if not of the above results too, which avoids the use of Fourier transforms of tempered distributions.

(b) It is clear from the above results that the correct range for $p$ in which to look for linear independence theorems for functions in $L_{p}\left(\mathbb{R}^{n}\right)$ is $1 \leq p \leq 2$ if the result is to be independent of $n$. For which locally compact (Lie) groups is it true that all non-zero functions in $L_{p}\left(\mathbb{R}^{n}\right)$ for $p=1$ (or $1 \leq p \leq 2$ ) have linearly independent translations?

\section{Acknowledgement}

I want to thank A. Carbery for pointing out references that led me to Hörmander's article. 


\section{References}

[1] G. Edgar and J. Rosenblatt, 'Difference equations over locally compact abelian groups', Trans. Amer. Math. Soc. 253 (1979), 273-289.

[2] K. Guo, 'On the $p$-approximate property for hypersurfaces of $\mathbb{R}^{n}$, Math. Proc. Cambridge Philos. Soc. 105 (1989), 503-511.

[3] L. Hörmander, 'Lower bounds at infinity for solutions of differential equations with constant coefficients', Israel J. Math 16 (1973), 103-119.

\section{Mathematics Department}

Ohio State University

Columbus, $\mathrm{OH} 43210$

Current address:

Mathematics Department

University of Illinois

Urbana, IL 61801 